\title{
A Range Based and Range Free Localization in Wireless Sensor Network
}

\author{
C Bala Subramanian, M Maragatharajan, S P Balakannan
}

\begin{abstract}
Wireless Sensor Networks is an ever changing research area in the communication field. Sensor networks are crucial means for various application areas like acoustic detection, medical monitoring, military surveillance etc. Different research issues are encountered in that field often. Among them, Localization is a major issue-based concept in wireless sensor network (WSN) which needs lot of focus and remedial mechanisms. The identification of the location of the sensor nodes is a critical factor in data gathering in the sensing field. The problem faced in this area of application is identifying the physical being of the sensors their post- deployment. This paper aims at proposing certain strategies and schemes in resolving the issue of localization. The study aims at focusing the range based and range free localization techniques which may aid the current and future researchers in and technologists who intend to pursue learning of application in wireless sensor networks.
\end{abstract}

Keywords : Angle of arrival, Localization, Mobile anchor, Received signal strength, Time of arrival.

\section{INTRODUCTION}

$\mathrm{O}_{\mathrm{f}}$ late in there has been a heavy increase in the need for monitoring and sensing remote environment in technological applications. Wireless Sensor Networks (WSN) is one of the chief advancement in technological devices. WSN has become a highly dependable and feasible device in meeting the requirements and specifications of advanced communication and computing technology. In general, information are sensed and passed to the base station as sent by the sensor nodes. Usually, sensor nodes are formed out of a mote [5] having certain amount of sensor devices. In fact, every sensor node is said to possess a microcontroller, which does the processing of the sensed information. Additionally, the sensor node is allied with a memory unit which stores the received information, prior to sending to the base station. For discharging the process, the sensor node is aided by a power battery to derive energy source. There is a transceiver for the purpose of receiving / transmitting the

Revised Manuscript Received on December 05, 2019

* Correspondence Author

C Bala Subramanian*, Department of Information Technology, Kalasalingam Academy of Research and Education, Virudhunagar Dt., Tamil Nadu. India. Email: baluece@gmail.com

M Maragatharajan, Department of Information Technology, Kalasalingam Academy of Research and Education, Virudhunagar Dt., Tamil Nadu. India. Email: maragatharajanm@gmail.com

S P Balakannan, Department of Information Technology, Kalasalingam Academy of Research and Education, Viruthunagar Dt., Tamil Nadu. India. Email: balakannansp@gmail.com signals from / to other nodes. Sensor nodes are fitted with an ADC which converts the analog signals to digital signals.

In practical terms, it is feasible to attach any sort of sensor devices with a mote. This feasibility enhances the utility of sensor nodes in various critical and crucial applications. Some of such applications include surveillance of military bases and activities and movement of human around protected and restricted environments. Sensor devices are ideal in case of warning issued regarding forest fire and smoke emission detection. It is to be noted that sensor nodes can either be mobile or static in their nature depending upon the utility specifications. Equally significant is that sensor nodes are chosen at random basis whereby there is no mandatory for specified network topology. Basically, once the information is passed to the base station, the local information enables the relevant identity or locating such information. Localization is generally an issue related to locating the existence of sensor nodes in the specified regions[5]. This is necessary due to the demand of processing the sensed information in the context of geographical locations where the sensor nodes could be installed. Of late, GPS has become a major source of location identification process under sensor nodes processing, yet with certain constraints [1].

i. Providing GPS back up to all types of sensor nodes incurs heavy investment costs.

ii. The disproportion with regard to the size of the sensor nodes with that of GPS, as the sensor nodes will be normally bigger.

iii. When the sensor nodes are prone to even minor damage, then there is a risk of shedding more money for GPS.

iv. Battery will lose its longevity when GPS is fitted with every sensor node.

As these constraints are inevitable and equally manageable, anchor nodes come as alternative. This anchor node is much like a sensor node being able to be static or mobile and does the functions of a sensor node yet with GPS fitted with it. However, cost wise, anchor node too is expensive. When the anchor nodes are installed within the radius of the similar areas of sensor nodes, there is a possibility of transmission of beacon messages. These beacon messages help the sensor nodes in identifying or locating the geographical locations. 
When localization is a serious concept to be discussed, it comes under two different categories namely range based or range free. Under range based localization method, an exclusive ranging hardware is used to measure the distance between nodes. In addition, there is a need for standalone hardware such as TOA for measuring the time of arrival, AOA for angle of arrival, TDOA for measuring the time difference of arrival and RSS in measuring the received signal strength. Such time differences undergo conversion into measurements under distance pattern. This type of measurement scheme is bound to provide accuracy but at the exorbitant cost factor. With regard to range free scheme, measurement of distance is made under the constraints reflected by the geographical location of the mobile anchor node. Range free scheme is less expensive as it does no require any sort of hardware. Range free scheme undertakes measuring the distance by availing large quantity of messages but does not offer accuracy as much as the range based scheme does. Despite these shortcomings, range free schemes are largely opted due to their cost efficiency and less complexities with regard to their implication. This paper aims at focusing various pros and cons of range based localization and range free localization schemes.

\section{RANGE BASED SCHEMES}

This section deals with various ranging techniques prevailing in the current state of technological applications.[10]

Time of arrival (TOA): In this approach, the measurement of distances related to reference points is done by the differences occurring in time in terms of signals propagation. Usually, the signals are transmitted by sensor nodes to the neighbor nodes, under a said velocity, for which the neighbor nodes respond with a signal. The time of arrival (TOA) is measured by way calculating or measuring the time differences occurring between the transmission and receiving of signals. If the estimation is expected to face minimized error, then the speed of propagation should always be less than ultrasound, prevailing in wireless scheme.

Angle of arrival (AOA): Under this scheme, individual sensor node is provided with more than one microphone and a speaker. The receiving node is capable of determining the angle of the transmitted signal based on the direction by which the transmission takes place. If there are more microphones in a single sensor node, then all these microphones can listen or hear the transmitted signal. As the arrival of transmission signal varies in several nodes, the difference of arrival determines the angle of arrival. This purports to the fact that the size of the sensor nodes will differ with respect to the number of microphones fitted in each sensor node.

Time difference of arrival (TDOA): When signals reach the individual sensor node, the difference in terms of arrival is calculated. As the sensor nodes are equipped with speaker and microphones, the sensor nodes can easily listen the arrival of signals and can easily record the time of arrival. Simultaneously, the recording of time chirps pattern, produced from the speaker also takes place. This record of differences in time is essential in calculating the position of every node. Nevertheless, there is a possibility for the occurrence of inaccurate recordings due to line-of-sight issues.

Received signal strength (RSS): The determination of the distance between the transmitter and the receiver is based on the difference applicable in terms of the power in transmitted and the received signal. Here, it is better to remember that according to path loss model, there is a decrease of the strength of a signal in proportion to the distance involved. Of these four discussed models, RSS has an edge over other three as it does not involve any hardware additionally. RSS is less expensive also as it utilizes the built-in transceiver in the wireless sensor node.

\section{A. Dynamic Fine-Grained Localization}

Savvides.A [13] came out with a novel propagation of location identity scheme called AHLoS. Initially, any sensor node can identify only a fraction of its location. As an extended process, other nodes calculate their location or position by way of using distributed algorithms. For this algorithm, the information is to be derived from the location aware nodes, otherwise called anchor nodes. In practice, there are two phases involved in determining the geographical location of a node. Of these two phases, the first is called the ranging phase which does the estimation of the distance between the location aware nodes and the other nodes. In this paper, two ranging techniques are adopted viz. received RF signal strength and, ultrasound based. For the sake of countering and overcoming the inconsistency with regard to RSSI, a model is applied, which is derived on the power level of each node.

$$
P_{\text {RSSI }}=\frac{X}{r^{n}}
$$

where, ${ }^{P_{\text {RSSI }}}$ represents the register reading of RSSI, ${ }^{r}$ refers the distance between two nodes, ' $\mathrm{X}$ ' and ' $\mathrm{n}$ ' are functional constants derived from function of ' $r$ '. As mentioned in the previous context, there could be variation in the measurement of signal strength. This is prevented by using the time difference of transmitted signals and ultrasound signals arrival. By this, the distance between two nodes is measured. When the measurements were compared, it was found that the ultrasound based on TDOA proved to be ideal and convenient in localization of fine grained nature. This is due to the factor that ultrasound based are not prone to any damage against physical factors. The current study engaged implementing the ultrasound equipped sensor nodes named Medusa.

The next phase, location estimation phase, involves the combination of range measurements and the information on location of known nodes. This combination process enables in determining the geographical position of the sensor nodes. This technique is termed as iterative multi-lateration, by which the limitation of the number of location aware nodes in the sensing fields can be effected. 


\section{B. Multilateration Primitive for Node Localization Problems}

Savvides.A [14] exhibited a collaborative natured algorithm called multilateration algorithm, for determining the geographical location of any node in a sensor network. This is necessitated since the nodes can be positioned far away, probably several hops, at a far off distant location. When there is a need to measure the distance between an anchor node and other sensor nodes, ranging techniques are employed. Quite naturally, this process involves range based scheme in addition to second generation Medusa nodes filled under ultrasonic sensors. Through this approach, localization can be effectively exploited. As it is said, collaborative scheme displays, the localization or the determination of the sensor nodes position is feasible when nodes which are not in direct connection with anchor nodes and sensor nodes collaborate.

There are three phases under this collaborative multilateration algorithm, namely forming of collaborative sub-trees, initial estimation of computation and, refinement of position. Under the first phase, there is a collaboration between the unknown sensor nodes and the anchor node to form a sub tree. It is this sub tree which asserts the fact that there is a unique means for every unknown node to determine its position. The second phase involves the calculation of the initial location by the unknown sensor nodes. This happens out of the measurements made among the inter-nodes and the location aware anchor nodes. In the third phase, least square computation schemes are employed in estimating the position of the sensor nodes for the purpose of refinement.

In general, collaborative multilateration is noted for its feature and characteristics of being centralized and distributed. Of the three phases discussed, the first and the second are able to operate on parallel since they do not depend each other. The third phase comes to the operation only after the two phases and is able to withhold at any given time based on the need or specification of the application. However, in centralized computation, once the unknown sensor nodes determine the estimation of location the third phase ceases to act. On the other hand, in distributed computation, the third phase withholds based upon the sufficiency of estimation of position or requirement of further results. In this current attempt, Kalman Filter position refinement.

\section{Ad-Hoc Positioning System (APS)}

Niculescu.D [10] proposed a method by which the nodes are let to collaborate in order to identify the location and node orientation. The method has an assumption that only a fraction of nodes is conscious of its geographical location. Here, the capacity of the nodes in determining the sense of the direction of the received signal plays a significant role in localization. This method is based on range based AOA metric in determining the position of the sensor nodes. In case of measuring the angle of received signals, there must be antenna or ultrasound receivers enabling the nodes. Nevertheless, there is another process which involves ad hoc network, whereby ad hoc positioning system (APS) algorithm has been derived. Yet, the major method is employed for solving the optimization problem of

constraint with the ad hoc network is that it can effect communication among the neighbor nodes only. So, in this paper, an assertion is made to discuss forwarding of orientation information from anchor nodes, otherwise called location aware nodes. Functionally, Ad hoc positioning system(APS) is composed of a combination of distance vector routing (DV) and beacon based positioning (GPS). It is known that there are some sensor nodes not in direct contact with the anchor nodes.

The purpose of forwarding the orientation information is that such nodes get the possibility to determine their location with the corresponding position of the anchor nodes. The paper dealt with two more algorithms viz. DV- Bearing in which bearing is deemed to be an angle in connection with an object, and DV-Radial wherein radial is deemed to be a reverse of bearing. of these two algorithms, DV-Bearing facilitates each of the sensor nodes in getting the due information from the anchor node. Similarly, DV-Radial lets the nodes to access radial and bearing information from the anchor node. The immediate neighbor nodes of anchor node are able to compute their location once they receive the orientation information of the anchor node. On the other hand, the location computation for other sensor nodes is carried out by forwarded orientation information.

\section{Adaptive Path Planning}

Kim.K [8] came out with a scheme involving adaptive path planning in terms of mobile anchor node. In this method, the location of the node is estimated by measuring the received signal strength treating it a ranging technique. By this technique, the distance between an anchor node and the sensor node is measured. Comparatively, the mobile anchor range is measured as twice than the range of sensor node. Within the mobile anchor, in this paper, there is a division such as ground and aerial anchors, so that the ground anchor node is capable of traversing on the surface and the aerial anchor node is capable of flying on air by reaching far-fetched and remote areas.

The localization of the sensor node of the proposed scheme comes under three phases namely reference movement, sensor localization, and movement path decision. Initially, at the first phase, a regular triangle is formed around a sensor node when the position of three beacon points is determined by an anchor node. By this way, beacon messages and the initial location of the beacon points to the sensor nodes are transmitted. This enables the determination of the location of the sensing node by the sensor node. When there is a need for increase in the number of location aware nodes, there is a recursive transmission of beacon messages, using range check technique. In this range check technique, the limit of the hop count is set as two and there has been a considerable reduction of the anchor traversal length and the required number of beacon messages for localization.

\section{E. Dual RSSI Trend Based Localization}

Pratap Kumar Sahu [11] discussed a localization scheme, based on various RSSI values, derived from the anchor nodes. For determining the maximum strength of the signal, polynomial model is applied between the distance found between the mobile anchor and

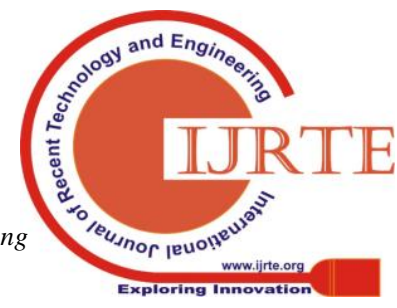


the sensor node on one side and, the RSSI values on the other side. The location of the sensor node is tracked at the intersection of the perpendicular lines pointed from the two trajectories. This method has a major advantage in the way of not requiring any absolute RSSI values. In general, the RSSI value is calculated using this formula

$$
R S S I(d)=P_{r}-P_{L}\left(d_{0}\right)-10 \eta \log _{10} \frac{d}{d_{0}}+X_{\sigma}
$$

where $\mathrm{d}$ is the distance, $P_{r}$ represents the transmission power, $P_{L}\left(d_{0}\right)$ refers the path loss with $\eta^{\prime}$ being the path loss exponent. RSSI is based on the model of guassian variable $X_{\sigma}$ and values of $\eta$ and $\sigma$ depend upon the environment to which it is propagated.

\section{F. Density-Based Outlier Detection}

Khalid K. Almuzaini [7] exploited the localization technique of density based outlier detection (DBOD) from data mining. This mechanism involves picking the k-nearest neighbors for the sake of sensor nodes and is distinct from the conventional measurement approaches regarding the nodes. DBOD facilitates the densities prevailing in every point enabling the calculation of the means of these points. The candidate points are identified from the points of greater densities than the mean. Ranging technique is applied in measuring the distance between the unlocalized node and the anchor node, thereby the sensor is surrounded by radii. In this manner, a set of points is formed by the intersection of the imaginary circles while the sensor's location is determined by way of the derived mean points. For the sake of accuracy in this regard, generalized geometry of dilution precision (GGDP) is depended by making use of the formula,

$$
\mathrm{r}_{m}=\frac{\varphi_{m}}{\gamma_{m}^{2}}
$$

where,

$$
\gamma_{m}=\sum_{i=1}^{m} \frac{1}{\sigma_{i}^{2}} \text { and } \varphi_{m}=\sum_{i=1}^{m} \sum_{j=1, j>1}^{m} \frac{\sin ^{2}\left(\alpha_{i}-\alpha_{j}\right)}{\sigma_{i}^{2} \sigma_{j}^{2}}
$$

where $\sigma_{i}^{2}$ is the Gaussian distribution of distance error of node ${ }^{i}, \alpha_{i}$ represents the angle orientation of $\mathrm{i}^{\text {th }}$ anchor node and ${ }^{m}$ refers the number of anchor nodes and location aware nodes. Ultimately, it can be asserted that there is an increase of GGDP value in proportion to the decrease of localization.

\section{RANGE FREE SCHEMES}

\section{A. Localization of Wireless Sensor Networks with a Mobile Beacon}

Sichitiu.M.L [16] deals with a localization algorithm devised on the basis of Bayesian inference. As is known, each of the unknown node is said to be in direct contact with the mobile beacon. The approach involved use of a single beacon node which can traverse around the sensing environment and involved ranging by using received signal strength(RSSI). A salient feature of this approach is that there is no need for any extended hardware and is easily available in electronic markets. Once the deployment is over, there is a traverse of the sensing field by the mobile beacon followed by the broadcast of beacon packets. On such occasions, the unknown nodes can easily identify them as the beacon packets meant for the specified range of mobile beacon. Simultaneously, RSSI is measured in each of the unknown

nodes which along with the coordinate position of the beacon put together determine the geographical location of the sensor nodes. Localization is carried out by way of considering the trajectory of the mobile beacon, and such trajectory is expected to be close for delivering accuracy in localization of the unknown nodes. Nevertheless, for the sake of attaining accurate computation or its improvement, an initial calibration phase is effected prior to deployment.

\section{B. Path planning of mobile landmarks for localization}

Koutsonikolas.D [9] gave account of three path planning algorithms, to define the mobile beacon node's movement in the sensor network. The localization algorithm dealt with in this paper is multihop localization algorithm, by which sensor nodes determine their location by broadcasting beacon messages to other sensor nodes. This method enables even far off and remote nodes from the mobile beacon too can localize their positions. The mobile beacon retains its initial position once it completes it traversal act on the sensing field. This necessitates mobile beacon reconfiguration in starting the localization repeatedly as there is an estimation of the position. Such a situation is common vicinity in mobile scenario which pertains to the provision of a localization epoch number for each beacon. As a result, there is a comparison of the new epoch number with the previous in each sensor. When the comparative result shows new epoch number to be greater, then a reset is affected for the position estimate, followed by renewed localization. It is to be noted here that as the localization gets fresh start, a probability distribution function (PDF) chart is devised within the calibration phase. From this chart, the list of all nodes and their corresponding RSSI values can be referred.

\section{Anchor-Guiding Mechanism for Beacon-Assisted Localization}

Hang.C.T [2] came out with a proposal for a mechanism on mobile anchor node for deriving efficient localization. The approach attempted in identification of the node's location by adopting the range free scheme involving geometric constraints. Through the beacon message, the sensor node is capable of identifying the presence of an anchor node within the communication range. Once the location of an anchor is defined, then the sensor believes itself to be within the communication range, which is also called as estimative region. This paper proposed four phases related to localization and identification which are promising region, weighting phase, beacon locations selection and phase construction phase. As for the first phase, the promising region is determined by both estimative region and the communication range together. The formula used to calculate the promising region is

$$
P R_{s, t}=\left[\left(x_{s, 1}-r, y_{s, 1}-r\right),\left(x_{s, 2}+r, y_{s, 2}+r\right)\right]_{\mathrm{t}}
$$

where $\left(x_{s, 1}, y_{s, 1}\right),\left(x_{s, 2}, y_{s, 2}\right)$ represent the coordinates of the estimative region and ${ }^{r}$ refers the communication range.

This is one way an input for the second phase in which promising region undergoes division into grids. Subsequently, weights of these grids are estimated individually. 
The third phase involves in attaining the accuracies of localization by the positions of the mobile anchor broadcasting beacons take place. These regions tend to be called as the most promising grids. The final phase involves the creation of the shortest path for traversing through the promising grid. All these four phases enable the purpose of overcoming the inaccuracies occurring in localization.

\section{Perpendicular Intersection}

Zhongwen Guo [19] describes range free localization scheme called perpendicular intersection (PI) which employs mobile features. Through this scheme, the location of a node can be computed by way of geometric constraints of perpendicular intersection. When direct mapping of absolute RSSI values to distances are kept off, there is a possibility to overcome any errors. Rather, the scheme proceeds with the use of RSSI values measures from mobile beacon to the sensor nodes. In the process, beacon packets are broadcast when there is a traversal of mobile beacon around the sensor node. Subsequently, there is a projection of the trajectory as the sensor node receives the greatest RSSI value. It gives way for determination of another projection around the sensor node. This is to draw a perpendicular out of these two projections interjecting at the centre so as to locate the sensor node. The final outcome is a virtual triangle (VT) when these two trajectories are combined. Sensor nodes normally prefer two pairs of beacon points in determining the localization and in certain cases, there may be requirement of more than two pairs of beacon points.

\section{E. Localization with Mobile Anchor Points}

Ssu.K.F [16] illustrated the concept of range free mechanism for localization of senor nodes with the adoption of a mobile anchor. The mechanism works on the basis of the geometric conjecture known as perpendicular bisector of the chord. The transmission range of the sensor node $(\mathrm{R})$ is deemed to be the circle of which the centre is deemed to be the sensor node's position. When the mobile anchor starts moving around the sensing environment, the beacon messages are broadcast allied by the information of the location. As the sensor node happens to hear the beacon message, it sets the position to be the beacon point. Similarly, within the communication circle, there are four beacon points for every sensor node on the circumference. It is only in this circle that two chords are drawn by making use of three different beacon points. This is to locate the sensor node, which is done when the perpendiculars drawn from the chords form an intersection of the circle at the centre. There will be an indication of error in case the length of the chord is short, which necessitates a definition of the threshold. The threshold $\mathrm{N}$ must fall within this range $0<\mathrm{N} \leq 2 \mathrm{R}$.

\section{F. Path Planning Algorithm for Mobile Anchor-Based Localization}

Chia-Ho Ou [3] referred the algorithm meant for localization, using a single mobile anchor. The proposed algorithm involves use of SCAN algorithm to denote the traversal of the anchor node, by which the localization of the sensor node can be made. Under normal conditions, in order to derive more beacon points a sensor node gets a chance to move along each other sensor nodes to a maximum of three times. The resolution of anchor's trajectory $\mathrm{R}-\mathrm{X}$ is the

distance between the two vertical line segments where $\mathrm{R}$ the communication radius and $X$ falls between 0 and $R / 3$. The length of the trajectory (D) of mobile anchor is calculated by this formula.

$$
D=(L+2 R) *\left(\left[\frac{L+2 R}{R-X}\right]+1\right)+(R-X) *\left[\frac{L+2 R}{R-X}\right]
$$

Detouring mechanism comes to the rescue of hindrances related to the sensing field.

\section{G. Obstacle Based Range-Free Localization}

Swapna Kumar.S [17] attempted at minimizing the localization accuracies in the sensing fields, caused by certain hitches and hindrances. The author came out with a convex optimization mechanism along with an attempt on diversification of sensor network's topology in terms of localization. With regard to anisotropic network, the distance between the sensor node and the anchor node is determined by classifying the sensor nodes. The mechanism employs two different inputs, of which the first one is related to propagation of beacon messages all through the sensor network. By the second approach, the beacon messages are broadcast to the neighbor nodes alone. The combination of the results of these two determines the position of the sensor.

\section{H. A Distributed Range-Free Localization}

Dil.B.J [4] attempted and proposed the COM-LOC++ localization algorithm for improving the COM-LOC distributed range free algorithm. COM-LOC makes use of the information which single anchor node sent for estimating the distance. On the other hand, COM-LOC++ enhances the performance level by adopting all the information received from all the anchor nodes. The proposed scheme and method is capable of reducing the error rate by $82 \%$. Though the scheme is RSS based, it comes under the category of range free. This is because it makes use of the difference measured between the transmitted and received signal but not the absolute RSS values. The proposed scheme makes use of the log normal shadowing method by which the signal strength over distance is modeled.

$$
P_{d}=P_{d_{0}}-10 n \log _{10}\left(\frac{d}{d_{0}}\right)+X_{\sigma_{d B m}}
$$

where $P_{d}$ represents signal power at distance $d, n$ refers the path loss component, $X_{\sigma}$ stands for the error of log normal shadowing. In addition, information from all the anchor nodes are considered and taken into account for estimating the hop distance for a single anchor node. When the hop count is set as 1 , then there is a forwarding of information by the anchor node to immediate neighbors. There is an increase of hop count value whenever there is a forwarded message. The algorithm is improved by COM-LOC++ with the inclusion of two phases called prediction phase and filtering phase.

\section{Multihop Range-Free Localization}

Qingjun Xiao [12] discussed the localization based on a pattern driven localization algorithm. The patterns are derived out of the distribution of hop count in anisotropic field. Out of these many patterns, three are discussed here which 
are concentric ring $(\mathrm{CR})$, centrifugal gradient $(\mathrm{CG})$ and distorted gradient (DG). Under CR, the accurate distance between the sensor and the anchor node is estimated by using CrMCs technique. In $\mathrm{CG}$, the variations in hop size are compromised or least considered by using Difftriangle. Sensor nodes exhibit their capability in assuming the distinguishing anchor messages and beacons out of these three patterns. The estimates regarding the distance are derived, by way of employing weighted multialteration, so as to locate the sensor's position. Thus, a clear demarcation of this process ends up with three phases namely, anchor classification phase, distance estimation phase and location estimation phase.

\section{J. A localization scheme with Dual Mobile Beacon}

Zhenbo Shi [18] proposed a novel scheme for range free localization of sensor nodes. The author aimed at dealing with two anchor nodes being positioned at equi-distance from each other. It is to be highlighted that these two anchor nodes move at similar speed yet by having different communication range. There could be little amount of message exchanges between the anchor node and the sensor node. The mobile anchors are liberal to move along either vertically or horizontally across the sensing field in the center. The anchor node engages in periodical broadcasting of the beacon messages. As each of the sensor nodes is capable of receiving beacon messages from mobile anchors, the distance between these two nodes, the anchor node and the sensor node, is measured. The sensor node sends an ACK once it receives the first beacon message from the second anchor. Every beacon message includes information such as the time of the message from both anchors, the coordinates of the messages and the list of neighbor nodes. These information and the geometric properties of a triangle are required to estimate the side of the sensor nodes.

\section{K. Double-Radius Localization}

Jijun Zhao [6] described a working principle on resolving the inaccuracies of the typical centroid algorithm for localization. This centroid algorithm envisages three kinds of increases - increase in the number of anchor nodes, increase in the number of circles for communication, and the increase in cost. The author aims at increasing the number of communication radius by still retaining the limited number of anchor nodes. The scheme, as the name indicates, Double-Radius localization algorithm, involves formation of dual radii circular communication region. Different forms of communication radii are the results of equally different signal ranges emitted by the anchor node. The inaccuracy can be avoided or removed if there is a smaller range of the intersection range from these circles. This smaller region could be identified or attained by partition judgment, despite the division of the region into number of divisions by the communication radii around the sensor node. In turn, the sensor node can identify its location or region around it, when the power of signal is exploited by the judgment mechanism. All these attempts ease the calculation of the centroid, for the purpose of smallest region. Such an approach is applicable for each sensor node to locate the smallest region and calculate the centroid. This is because the centroid thus calculated is the accurate location of the sensor node.

\section{CONCLUSION}

Sensor localization is treated as the most significant and essential case in the field of network management and network operations. As of now, it has been in practice to install or deploy the sensors without assessing the location to be preferred. Actually, localization should be done prior to deployment and currently there has been no technological assistance in locating them. Such is the case in current world scenarios. This paper aims at focusing and highlighting various range based and range free localization techniques related to wireless sensor networks. It is hoped that this attempt will enable more information for similar researchers and the technologists to review their application process. In all, this study will be an eye opener for those who are new to the wireless network field.

\section{REFERENCES}

1. Amitangshu Pal, "Loclization Algorithms in Wireless Sensor Networks: Current Approaches and Future Challenges," Network protocols and algorithms, vol 2, no.1,1943-3581, 2010.

2. Chang.C.T, Chang.C.Y, and Lin.C.Y, "Anchor- guiding mechanism for beacon-assisted localization in wireless sensor networks," IEEE Sensors J., vol. 12, no. 5, pp. 1098-1111, May 2012.

3. Chia-Ho Ou and Wei-Lun He, "Path Planning Algorithm for Mobile Anchor-Based Localization in Wireless Sensor Networks," IEEE Sensors J, vol. 13, no. 2, Feb 2013.

4. Dil.B.J, Havinga.P.J.M," COM-LOC++:A Distributed Range-Free Localization Algorithm in Wireless Networks," Sixth International Conference on Intelligent Sensors, 2010.

5. Gowrishankar.S, T.G.Basavaraju, Manjaiah D.H and Subir Kumar Sarkar ," Issues in Wireless Sensor Networks," Proceedings of the World Congress on Engineering, Vol I, July 2008.

6. Jijun Zhao, Hua Li, Zhiyuan Tang, Xiang Sun,"Research on WSN Double-Radius Localization Algorithm Based on Partition Judgment Mechanism," Wireless Sensor Network, vol 2, 639-644,2010.

7. Khalid K. Almuzaini, Aaron Gulliver, " Range- Based Localization in Wireless Networks Using Density-Based Outlier Detection," Wireless Sensor Network, vol. 2, 807-814, Oct 2010.

8. Kim.K, Jung.B, Lee.W, and Du.D.Z, "Adaptive path planning for randomly deployed wireless sensor networks," J. Inf. Sci. Eng., vol. 27, no. 3, pp. 1091-1106, May 2011

9. Koutsonikolas.D, Das.S.M, and Hu.Y.C, "Path planning of mobile landmarks for localization in wireless sensor networks," Comput. Commun., vol. 30, no. 13, pp. 2577-2592, Sep. 2007.

10. Nasipuri.A and Li.K, "A directionality based location discovery scheme for wireless sensor networks," in Proc. ACM Int. Workshop Wireless Sensor Netw. Appl., pp.105-111 Sep. 2002.

11. Niculescu.D and Nath.B, "Ad hoc positioning system (APS) using AoA," in Proc. IEEE Joint Conf. IEEE Comput. Commun Soc., pp. 1734-1743,Mar. 2003

12. Pratap Kumar Sahu, Eric Hsiao-Kuang Wu, Member, IEEE, and Jagruti Sahoo, "DuRT: Dual RSSI Trend Based Localization for Wireless Sensor Networks" IEEE Sensors J, vol. 13, no. 8, Aug 2013.

13. Qingjun Xiao, Bin Xiao,Jiannong Cao, and Jianping Wang, "Multihop Range-Free Localization in Anisotropic Wireless Sensor Networks: A Pattern- Driven Scheme," IEEE Transactions On Mobile Computing, vol. 9, no. 11, Nov 2010.

14. Savvides.A, Han.C.C, and Srivastava.M.B, "Dynamic fine-grained localization in ad-hoc networks of sensors," in Proc. ACM Int. Conf. Mobile Comput. Netw., pp.166-179,Jul. 2001

15. Savvides.A, Park.H, and Srivastava.M, "The bits and flops of the Nhop multilateration primitive for node localization problems," in Proc. ACM Int. Workshop Wireless Sensor Netw. Appl., pp. 112-121,Sep. 2002.

16. Sichitiu.M.L and Ramadurai.V, "Localization of wireless sensor networks with a mobile beacon," in Proc. IEEE Int. Conf. Mobile Ad-Hoc Sensor Syst., pp. 174-183,Oct. 2004.

17. Ssu.K.F, Ou.C.H, and Jiau.H.C, "Localization with mobile anchor points in wireless sensor networks," IEEE Trans. Veh. Technol., vol. 54, no. 3, pp. 1187-1197, May 2005. 
18. Swapna Kumar.S, Dr Nanda Kumar.M, Dr Sheeba.V.S, "Obstacle based Range-Free Localization-Error Estimation for WSN," IJCSI International Journal of Computer Science Issues, Vol. 8, Issue 5, No 2, Sep 2011.

19. Zhenbo Shi, Yi Zhu, Ya nan Ni, Jia Shi and Shu Yan, "A localization scheme with Dual Mobile Beacon in Wireless Sensor Networks," Information Technology journal, vol.12, 2426-2432, 2013.

20. Zhongwen Guo, Ying Guo, Feng Hong, Zongke Jin, Yuan He, Yuan Feng, Yunhao Liu, "Perpendicular Intersection: Locating Wireless Sensors with Mobile Beacon," IEEE Trans. Veh.Technol., vol.59, issue.7 Sep2010.

21. Bala Subramanian C \& Balakannan S P, "Optimized Trajectory Planning For Mobile Anchors In Wireless Sensor Networks", IEEE Xplore, ISBN: 978-1-5090-4778-9, March 2017.

22. Renuka I and Bala Subramanian C, "Enhanced Route Planning for Mobile Anchors in Localization of WSN", IEEE Xplore, ISBN: 978-1-4799-3913-8, Page No. 649 - 652, May 2014.

\section{AUTHORS PROFILE}

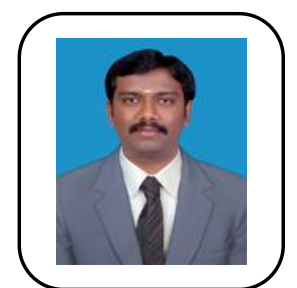

C Bala Subramanian received his Bachelor of Engineering in Electronics and Communication Engineering from Anna University, Chennai by 2006. He received his master of Engineering in Applied Electronics from Anna University, Chennai by 2008 . He is pursuing Ph.D degree Information Technology with Kalasalingam University in $\mathrm{He}$ is working as an Assistant Professor in the Department of Computer Science Engineering, Kalasalingam Academy of Research and Education. His areas of interest are Image and Signal Processing, Sensor Networks, Adhoc Networks.

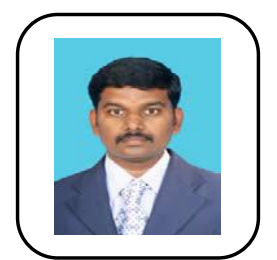

M Maragatharajan received his Bachelor degree in Electronics \& Communication Engineering from Anna University by 2007 . He has received his Master degree in Information Technology from Kalasalingam University, 2010 and he has completed his ph.D in the area of MANET. He has worked as a Project Associate in TIFAC CORE in

Network Engineering, Kalasalingam University from 2007 to 2008. Currently, He is working as an Assistant Professor in the Department of Information Technology, Kalasalingam University. His areas of interest are Ad-hoc Networks, Wireless Networks, and Network Security.

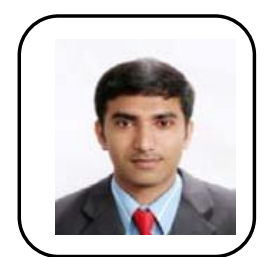

S P Balakannan received his Ph.D. degree from the Department of Electronics and Information Engineering at Chonbuk National University, South Korea (2010). He has received his master degree (5 years integrated) from the Department of Computer Science and Engineering, Bharathiar University, India, in the year 2003. He has worked as a Project Assistant in Indian Institute of Technology (IIT) Kharagpur, India from 2003 to 2006. Currently, he is working as Associate Professor in the Department of Information Technology, Kalasalingam Academy of Research and Education, Tamilnadu, India. His areas of interest include Wireless Network, Network Coding, Cloud \& Green Computing, Cryptography, and Mobile Communication. 